\title{
Traduire
}

Une autre perspective sur r tr traduction

Revue française de la traduction

$226 \mid 2012$

Face au miroir

\section{Les traducteurs de papier}

Traducteurs et interprètes dans la littérature québécoise

Jean Delisle

\section{(2) OpenEdition}

Journals

Édition électronique

URL : http://journals.openedition.org/traduire/136

DOI : $10.4000 /$ traduire.136

ISSN : 2272-9992

Éditeur

Société française des traducteurs

Édition imprimée

Date de publication : 1 janvier 2012

Pagination : 7-20

ISBN : 039-773X

ISSN : 0395-773X

Référence électronique

Jean Delisle, "Les traducteurs de papier », Traduire [En ligne], 226 | 2012, mis en ligne le 01 janvier 2012, consulté le 11 octobre 2020. URL : http://journals.openedition.org/traduire/136 ; DOI : https:// doi.org/10.4000/traduire.136 


\section{Les traducteurs de papier Traducteurs et interprètes dans la littérature québécoise}

\section{Jean Delisle}

Le texte ci-dessous est issu de plusieurs articles publiés dans Circuit, la revue de l'Ordre des traducteurs, terminologues et interprètes agréés du Québec (OTTIAQ), notre association sœur québécoise (1). L'équipe de Traduire les a resserrés et regroupés en un seul texte qu'elle est très heureuse de proposer à ses lecteurs avec l'aimable autorisation de l'auteur, M. Jean Delisle, et de Mme Betty Cohen, directrice de Circuit.

"Antoine, le traducteur, n'avait pas seulement traversé le roman, il l'avait vécu, réellement, avec toutes les fibres de son être. Tout à coup, il comprit. II comprit qu'il était lui aussi un personnage de fiction, que tout cela, réalité, fiction, se fondait en un seul et même rêve. "

Nicole Lavigne

(Moscou la nuit, 1998)

Nombreux sont les écrivains d'expression française au Canada qui ont trouvé dans la pratique de la traduction un moyen de subsistance connexe à l'écriture originale. "Nos écrivains, a écrit Pierre Baillargeon, exercent tous un métier parallèle ; ils sont avocats, médecins, traducteurs, prêtres " (Baillargeon, 1944 : 53). Dans son roman Les Médisances de Claude Perrin, Pierre Baillargeon écrit encore : "Manger ou ne pas manger, là est la question. Je soutenais que, pour l'écrivain, il y a toujours de la vache enragée, le petit emploi [...] " (Baillargeon, 1973 : 118). À ses yeux, le métier de traducteur est un "petit emploi ».

(1) "Les traducteurs dans la littérature québécoise ", Circuit, $n^{\circ}$ 78, 2003, p. 20-21; " Les traducteurs de moins en moins fictifs ", Circuit, $n^{\circ}$ 79, 2003, p. 25-26; " Les conditions de travail du traducteur fictif ", Circuit, $n^{\circ}$ 80, 2003, p. 30-31 ; " Les traducteurs de papier : un portrait réaliste ? ", Circuit, n 81, 2003, p. 18-19. 
Les auteurs ayant consacré à la traduction une part importante de leur activité intellectuelle ont-ils transposé dans leurs œuvres de création leurs préoccupations de traducteur ? S'ils ont mis en scène des traducteurs fictifs, ceux-ci leur ressemblent-ils ? Quel portrait en ont-ils tracé ? Les ont-ils représentés au travail, entourés de leurs dictionnaires et aux prises avec leurs problèmes d'équivalences ? Nous renseignent-ils sur leur âge ? leur état civil ? leur niveau de scolarité ? Le personnage-traducteur est-il un dilettante ou un salarié ? Où travaillet-il ? dans une administration publique ? une entreprise privée ? une agence de publicité ? une maison d'édition ? une salle de rédaction ? Quel regard porte-t-il sur son métier ? Est-il politisé ? En somme, le portrait du traducteur qui se dégage de la littérature québécoise estil conforme à celui du traducteur non fictif?

$\mathrm{Si}$ « le roman est un document social ", comme le prétendait Alfred Desrochers (cité par Maurel 1946 : 267), nous devrions nous attendre à ce qu'il y ait coïncidence plus ou moins grande entre le " traducteur de papier " et le " traducteur en chair et en os ". II n'est pas impossible non plus que l'image du traducteur évoluant dans l'univers fictif soit investie d'une valeur proprement littéraire et qu'il soit intégré, consciemment ou non, au processus de création littéraire.

\section{Personnages-traducteurs}

Un premier constat s'impose : la matière à étudier ne manque pas. À ce jour, j'ai recensé pas moins de 225 écrivains-traducteurs et 136 personnages-traducteurs dans 86 œuvres littéraires appartenant aux genres les plus divers : romans, nouvelles, contes, pièces de théâtre et pastiches.

Avant de parler des traducteurs imaginaires eux-mêmes, disons un mot des auteurs qui leur ont prêté vie. Les écrivains ayant exercé le métier de traducteur à temps partiel ou à plein temps appartiennent à toutes les époques de l'histoire littéraire du Québec. Philippe Aubert de Gaspé (1786-1871) a été de 1813 à 1816 "Traducteur français et secrétaire français du Gouverneur en Conseil ॥. De 1841 à 1863, retiré dans son manoir de Saint-Jean-Port-Joli, il a mené une vie de patriarche solitaire et traduit des romans de Walter Scott. Plus près de nous, Lori Saint-Martin est une jeune auteure qui a habité Toronto où elle enseignait la littérature québécoise et la traduction, en plus de pratiquer le métier d'interprète de conférence. Elle vit aujourd'hui à Montréal et enseigne à l'UQAM. Dans son premier livre de fiction, Lettre imaginaire à la femme de mon amant (1991), une nouvelle intitulée "Départs " met en scène une interprète pigiste.

Les interprètes sont des voyageurs et des parleurs, ils savent traiter en deux ou trois langues de choses qu'ils ne comprennent dans aucune. Leurs bagages sont légers, ils peuvent recommander un hôtel à Whitehorse ou un restaurant à Manille, vous fourniront les coordonnées d'un charmant collègue d'Amsterdam. Ils aiment les mauvais calembours, les mots croisés, la distance. Hier ils étaient partis et demain ils repartiront, entre-temps ils parlent, sérieux, concentrés, polyglottes (Saint-Martin, 1991 : 31-32). 
En 2001, Marie-Célie Agnant, née à Port-au-Prince et vivant maintenant à Montréal, fit paraître Le Livre d'Emma. Un des personnages principaux de ce roman est une interprète, Flore, " une négresse ratée ", qui recueille les confidences d'Emma, internée dans un hôpital psychiatrique car elle est frappée d'une malédiction, comme le sont les femmes de sa lignée. L'auteure est elle-même traductrice et interprète culturelle et travaille auprès de communautés haïtiennes et latino-américaines.

Entre l'" ancien Canadien " et la néo-canadienne de la relève, bon nombre d'écrivains québécois ont touché à la traduction. Qu'il suffise de citer les noms d'Antoine Gérin-Lajoie, William Chapman, Jules Fournier, Alonzo Cinq-Mars, Rodolphe Girard, Harry Bernard, Pierre Baillargeon, Paul Morin, Jean Filiatrault, Jean Simard, Michel Garneau, Alfred Desrochers, Jean Hamelin, Alain Grandbois, Michel Beaulieu, Paul Chamberland, Madeleine Gagnon, Dominique Blondeau, Jacques Poulin, Hélène Rioux, Pauline Harvey, Jean Marcel.

\section{Métier : écrivain-traducteur}

On aurait tort de penser que les auteurs-traducteurs s'adonnent tous à la traduction littéraire. La plupart pratiquent ou ont pratiqué largement ce qu'on appelle la " traduction alimentaire ", bien que la traduction littéraire puisse être tout autant « alimentaire ". Ils sont pigistes pour des entreprises privées et des administrations publiques ou travaillent dans les services de dépêches des journaux. Le regretté Michel Beaulieu (1941-1985) m'a confié, par exemple, qu'il traduisait de la poésie pour son plaisir et, sous le couvert de l'anonymat, des romans pornographiques pour mettre du beurre dans les épinards...

Nombreux sont les écrivains québécois qui ont vécu et vivent de leur plume traductrice...

Dans un essai intitulé Le Romancier fictif, André Belleau a attiré l'attention sur l'abondance de romans québécois centrés sur un personnage-écrivain : plus de quarante entre 1940 et 1980. II était donc prévisible que les auteurs mettent aussi en scène des héros traducteurs qui " se trouve[nt] à l'extérieur de la littérature sans en être trop éloigné[s] ", note Belleau à propos de Marcel Larocque, personnage principal de La Fin des songes de Robert Élie (Belleau, 1980 : 82 , note 103).

Les 86 œuvres et les 136 traducteurs fictifs recensés jusqu'ici - et notre dépouillement n'est pas exhaustif - témoignent à l'évidence de la présence de la traduction dans l'univers littéraire québécois. Les deux tiers des titres recensés renferment au moins un personnage principal qui exerce le métier de traducteur ou d'interprète. Les autres contiennent des réflexions sur la traduction ou des personnages secondaires s'adonnant à la traduction. Ces chiffres seraient encore plus élevés si j'avais inclus dans mon étude les interprètes figurant dans les romans historiques qui ont pour cadre le Régime français. On y aurait vu défiler les deux interprètes 
de Jacques Cartier, les indigènes Dom Agaya et Taignoagny, la plupart des interprètes de Champlain ainsi que la majorité des interprètes qui se mirent au service des Français. J'ai délibérément exclu de mon corpus ces figures du Canada primitif, d'une part pour limiter l'ampleur de l'étude et, d'autre part, pour la centrer sur les écrits de "pure " fiction, ceux dans lesquels les auteurs sont le plus susceptibles de se projeter eux-mêmes par personnages interposés. Cette dimension autobiographique m'apparaît essentielle pour cerner cette espèce de « narcissisme inquiet " de l'écrivain québécois, selon l'expression de François Ricard (Blais, 1980 : 178). Autrement dit, le personnage-traducteur pourrait bien être le frère jumeau du personnage-écrivain par l'entremise duquel les auteurs cherchent à se définir eux-mêmes et à préciser le sens de leur activité en vue de se donner une conscience d'écrivain. Cette hypothèse est renforcée par le fait que bon nombre de traducteurs fictifs sont aussi écrivains(2), vivent dans l'entourage d'écrivains(3) ou souhaitent eux-mêmes écrire des romans(4).

\section{Traducteurs de papier}

II faut remonter à 1658 pour relever la toute première mention d'un traducteur fictif dans une œuvre de fiction littéraire autre qu'un roman historique. II s'agit en fait d'un interprète. Lorsque le vicomte d'Argenson, Pierre de Voyer, débarque à Québec en juillet 1658 pour y occuper le poste de gouverneur de la Nouvelle-France, les élèves du collège de Québec organisent une réception solennelle en son honneur et montent un petit drame trilingue français, huron, algonquin. L'un des personnages de cette pièce de circonstance est le "Génie interprète " qui traduit en français les passages déclamés en huron et en algonquin(5).

Jusqu'à la fin des années 1950, on dénombre relativement peu d'œuvres mettant en scène des traducteurs, moins d'une dizaine, dont Jean Rivard d'Antoine Gérin-Lajoie, La Maison vide d'Harry Bernard, Les Médisances de Claude Perrin et Commerce de Pierre Baillargeon ainsi que La Fin des songes de Robert Élie. Dans ces œuvres, le métier de traducteur apparaît comme

(2) Cf. Romain dans L'Homme foudroyé (D. Blondeau), Marie-Charles Craig dans Miss Charlie (S. Paradis), Claude Perrin dans Les Médisances de Claude Perrin (P. Baillargeon), Anne dans Une Histoire gitane (H. Rioux), le personnage anonyme dans N'entendre qu'un son (R.-B. Drapeau).

(3) Cf. Catherine dans Catherine I à V (J.-C. Clari), Michel Bullard dans Doux-amer (C. Martin), Sylvie Duchamp dans Opération orchidée (C. Villon), Théophile Brisefer dans Rue des Petits-Dortoirs (D. Bélanger), Carl dans La Séparation (J. Simard).

(4) Cf. Émir dans Élodie H. ou le juste retour des choses (L. Jolicœur), Gail Scott dans Héroïne (G. Scott).

(5) La Réception de Monseigneur le Vicomte d'Argenson par toutes les nations du païs de Canada à son entrée au Gouvernement de la Nouvelle-France, publiée par Pierre Georges Roy, Québec, imprimerie Léger Brousseau, 1890, 23 p. Cette pièce relève de ce que Beaudoin Burger appelle le "théâtre de collège " caractérisé par " [...] des "réceptions", des "actions", des tragi-comédies, des pastorales et des passions, mais aucune comédie [...]. Le répertoire est littéraire parce que les pièces sont écrites en vers et parce que leur signification repose sur des images, une parabole ou une allégorie [...] " (cité par Andrès, 1990 : 44). 
un pis-aller, une occupation exercée dans l'attente de trouver mieux. En raison de l'encombrement des professions libérales, Gustave Charmenil, dans Jean Rivard, se voit " obligé d'écrire pour les gazettes, de traduire, de copier, d'enseigner le français " (Gérin-Lajoie, 1947 : 119). Pour sa part, Claude Perrin avoue : "Finalement j'entrai comme traducteur dans une agence de publicité anglaise. J'acceptai cet emploi comme un tremplin pour parvenir plus haut " (Baillargeon, 1973 : 161). "Au bout de cinq ans, constate-t-il, je n'étais guère plus riche et j'étais devenu complètement bête " (ibid. : 166). Ce thème de l'abêtissement revient également dans La Fin des songes, où le traducteur Marcel Larocque se demande comment il peut "reprendre tous les jours ce travail idiot de traduction dans un journal " (Élie 1950 : 16), pour un " ridicule salaire " (ibid. : 61). D'humeur changeante et imprévisible, le solitaire Marcel affiche une attitude étrange. Des crises de " confusion de personnalités " (ibid. : 145) ponctuent la monotonie de sa vie jusqu'au jour où il se suicide en se jetant sous les roues d'un tramway.

À partir de 1960, le nombre de personnages-traducteurs se multiplie et on assiste à l'émergence de cette figure dans la littérature québécoise : plus d'une soixantaine de romans ou nouvelles présentent au moins un traducteur ou un interprète comme protagoniste. Deux romans en comptent jusqu'à neuf: Tuez le traducteur de Léa Pétrin et Sylvie Stone de Michel Beaulieu. Avec cinq chacun, Nata et le professeur d'Alice Parizeau et Visions de Jude de Daniel Poliquin ne sont pas en reste.

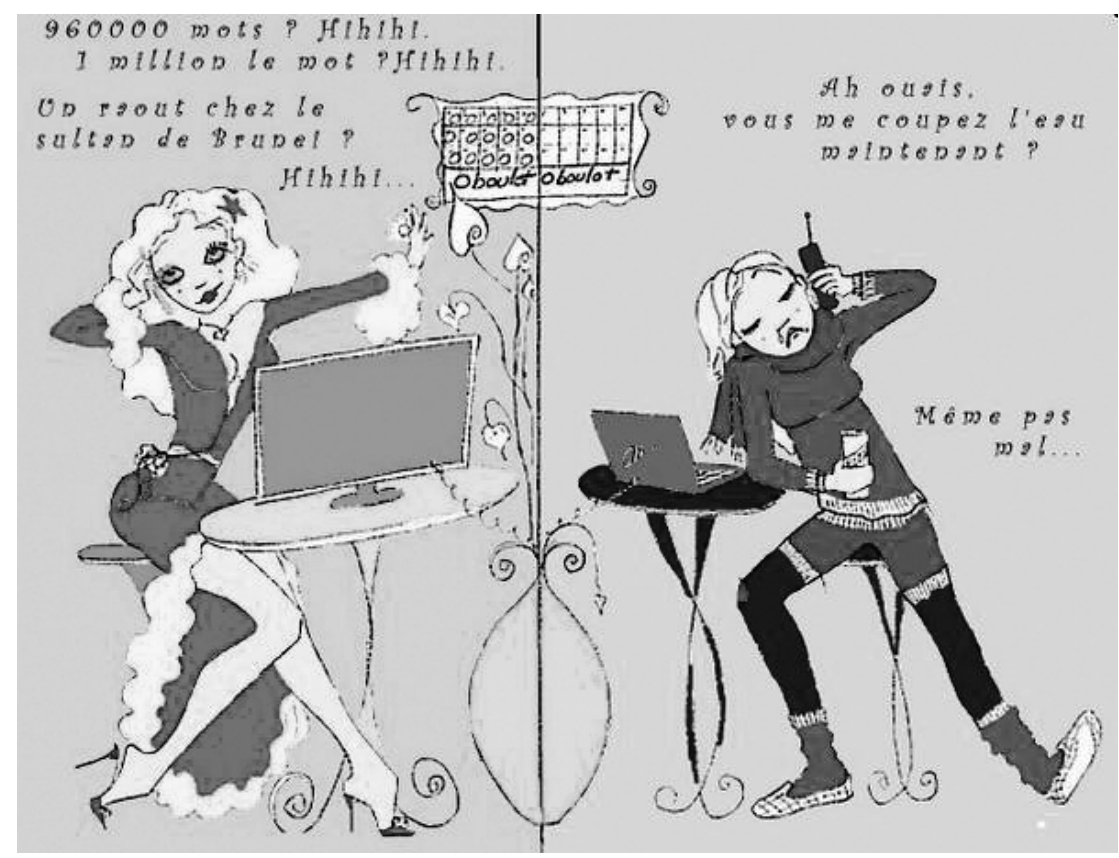

Illustration : Marlène Junius, http://alotoftralala.over-blog.com 


\section{Profil des traducteurs imaginaires}

Mais il y a plus important encore que le nombre de traducteurs dans les œuvres. Les auteurs québécois vont bien au-delà des clichés et des mentions superficielles qui servent habituellement à décrire les traducteurs fictifs en littérature. En scrutant les textes, on peut en effet obtenir des renseignements étonnamment précis sur le sexe de ces personnages, leur âge, leur état civil, leurs études, leur employeur et le genre de travail qu'ils effectuent.

Dans les œuvres dépouillées, on dénombre deux fois plus de traducteurs fictifs que de traductrices fictives, leur âge moyen est d'environ 35 ans (ce qui correspondait à l'âge moyen des membres de la Société des traducteurs du Québec en 1990), la majorité d'entre eux vivent seuls (ils sont célibataires, séparés, divorcés ou veufs) et ceux qui vivent en couple n'ont jamais plus de deux enfants. Parmi ces personnages figurent des journalistes, des écrivains, quelques professeurs, un ou deux commis, un apôtre, un pape, deux prêtres, un lion et quelques machines à traduire.

Le traducteur fictif est habituellement une personne cultivée qui détient un diplôme universitaire dans une des disciplines suivantes : droit, médecine, biologie animale, géographie, génie, lettres, linguistique, traduction. II traduit de tout : des catalogues de constructeurs automobiles, des annonces de corsets, des manuels de mécanique, Pétrarque, la Bible, des manuscrits persans, des dépêches, des bandes dessinées, les Débats parlementaires, de la propagande nazie...

Les traducteurs " réels " détiennent aussi des diplômes universitaires. Depuis 1973, une vingtaine de " sondages radiologiques " ont permis d'explorer les "replis de l'âme " des membres de l'Ordre qu'on a appelé "Estécois " jusqu'à ce que les traducteurs du Québec obtiennent la reconnaissance professionnelle et que la Société des traducteurs du Québec (STQ, d'où "Estécois ") se transforme en Corporation, puis en Ordre dans les années 1990. " En 1973, $56 \%$ des membres détiennent un diplôme universitaire et plus des deux tiers ont étudié la traduction. Treize ans plus tard, la proportion de diplômés universitaires atteint $83 \%$ et, parmi ceux-ci, pas moins de 23,4\% ont terminé des études supérieures de maîtrise ou de doctorat. En outre, le sondage de 1986 révèle que près de $39 \%$ des répondants ont fait des études dans des domaines non linguistiques, principalement en administration, en économie, en psychologie, en philosophie et en relations publiques " (Delisle, 1990 : 327).

\section{En pleine réflexion}

Les auteurs de romans ou de nouvelles mettant en scène des personnages-traducteurs nous renseignent non seulement sur ce que traduisent les traducteurs qu'ils inventent, mais ils nous les montrent souvent à leur table de travail. Que l'on songe, par exemple, au roman Les Grandes 
Marées, dans lequel Jacques Poulin décrit par le détail les instruments de travail de T.D.B. (Teddy Bear), retiré sur son île au milieu du Saint-Laurent :

D'aussi loin qu'il se souvenait, il avait toujours aimé les dictionnaires et les encyclopédies. Le Petit Robert, le gros Harrap's, le Grand Larousse, le petit Littré, le gros Webster remplaçaient les amis qu'il n'avait pas. [...] Malheureusement, il ne se souvenait plus s'il fallait traduire " knuckle ball " par " balle-jointure " ou bien par " balle-papillon " et il n'avait trouvé la solution ni dans le Harrap's, ni dans le gros Webster, ni dans le Grand dictionnaire d'Américanismes [sic] (Poulin, 1978: 17-18).

Mais Jacques Poulin va encore plus loin et nous entraîne dans les méandres de la réflexion du traducteur faisant la chasse aux équivalences. T.D.B. doit traduire une bulle dans une bande dessinée de Charlie Brown où il est écrit : "All conferences on the mound have been canceled until further notice ". La description de la réflexion du traducteur, qui tâte diverses solutions, occupe pas moins de quatre pages (Poulin, 1978 : 143-146). Avec lui, on a la satisfaction d'aboutir à la solution : " Aucune conférence au monticule jusqu'à nouvel ordre. "Comment, encore ici, ne pas voir sous les traits de T.D.B. Jacques Poulin lui-même, traducteur au journal Le Soleil de Québec, où il traduisit des bandes dessinées américaines?

Dans son premier roman, Hélèna, Claude Tatilon met en scène un romancier forcé par son éditeur de "pondre " quatre romans policiers érotiques par année. Ce "pisse-bouquins " est aussi traducteur pigiste d'annonces publicitaires. Le professeur Tatilon, qui enseigne, entre autres, la théorie de la traduction au Collège Glendon de Toronto, nous décrit, par l'entremise de son personnage, Philippe Joubert, les qualités d'une bonne adaptation publicitaire :

Je lui ai expliqué le pragmatisme de ce genre de traduction, où il s'agit beaucoup moins de se montrer fidèle à l'original que d'en produire un équivalent commercialement actif, c'est-à-dire un texte qui informe et qui accroche autant, sinon plus, que l'original. "Belle infidèle " ou plutôt "belle efficace ", adaptation plutôt que traduction (Tatilon, 1991 : 28).

C'est Claude Tatilon, pédagogue, qui sent le besoin d'illustrer cette règle de traduction au moyen d'un exemple (il s'agit d'une réclame de soutien-gorge) :

La traduction plate donnait ceci :

Un dernier coup d'œil à votre miroir et vous partez satisfaite. Le Doux \& Fin de Queen's galbe votre poitrine sans se laisser deviner.

Tulle élastique, $100 \%$ coton, sans armature, bretelles réglables, fermeture à deux positions. Existe en blanc, noir, ivoire et champagne.

Doux \& Fin

de

Queen's 
La traduction adaptée a finalement donné ceci :

Un dernier coup d'œil à votre miroir. Parfait. Sous votre robe légère, le Doux \& Fin de Queen's déshabille vos seins. Audacieusement.

Sans se laisser deviner, un souple liseré galbe leurs formes. Harmonieusement. Et son tulle élastique, ses bretelles réglables, sa fermeture à deux positions ajustent Doux \& Fin à vos mesures. Parfaitement.

$100 \%$ coton
disponible en blanc, noir, ivoire et champagne.
Doux \& Fin
de
Queen's

Dans Hélèna, le traducteur (l'auteur ?) nous présente donc sa conception d'une bonne adaptation publicitaire, le premier jet d'une traduction et la version finale (Tatilon, 1991 : 27-29). II ne manque, à vrai dire, que l'original anglais pour avoir le début d'un petit traité sur l'adaptation publicitaire...

Il arrive aussi qu'un écrivain transcende les difficultés ponctuelles de traduction et se laisse aller à des considérations d'ordre théorique sur la traduction, la compréhension ou le phénomène général de la communication. C'est le cas de René Lapierre, qui écrit à propos de la traductrice Gayle, dans Comme des mannequins :

Elle supportait facilement cette tension parce que le passage d'une langue à une autre avait pour elle quelque chose de naturel. Comme si comprendre une chose c'était d'abord la traduire, lui donner des sens équivalents : parler de la même chose en n'utilisant jamais les mêmes mots. Mais parler de quoi ? Parler pour qui ? À qui ? C'était ça le fond obscur, ce qu'il y avait précisément là de magnifique et de troublant, et qui lui échappait toujours. Les mots devaient défiler dans sa tête comme des pièces à conviction, des choses accumulables, comparables ; différentes mais semblables au fond (Lapierre, 1983 : 79-80).

Ce paragraphe résume l'essentiel de la problématique de la traduction : comment être Autre tout en restant le Même, comment être à la fois " différent "ET " semblable " ? Est-on sûr d'avoir bien compris le sens de ce qu'il faut traduire ou interpréter? Dans cette enquête en vue d'établir la preuve du sens, les mots sont bel et bien des " pièces à conviction ", comme l'écrit René Lapierre. En traductologie, les articles et les ouvrages savants traitant du processus de la traduction abondent. Faut-il s'étonner, dès lors, que l'universitaire (et peut-être traducteur?) René Lapierre évoque ces questions dans son roman ? II n'est pas le seul à discuter de la traduction d'un point de vue théorique dans une œuvre de fiction. Agnès Guitard, Jacques Poulin, Pierre Baillargeon(6), Jacques Brault, Nicole Brossard, pour ne citer que ces auteurs, consacrent aussi des pages lucides à cette question dans leurs œuvres de création. Ce faisant, ils font écho aux réflexions théoriques des traductologues, poètes et philosophes modernes tels que Berman, Bonnefoy, Meschonnic, Mounin, Ricœur ou Steiner.

(6) J'ai analysé la conception de la traduction de ce traducteur dans Portraits de traducteurs, p. 259-301. 


\section{Conditions de travail}

Les auteurs décrivent aussi en détail les conditions de travail des traducteurs fictifs. Cela était tout à fait prévisible. Je n'entrerai pas ici dans les détails, faute de place. Je me bornerai à dire ceci : que ces conditions de travail soient jugées bonnes ou mauvaises, les traducteurs de papier ne manquent pas de nous livrer leurs sentiments sur leur métier. Et il n'est pas défendu de croire que ces sentiments sont partagés par les auteurs eux-mêmes. Pour certains, la traduction est un refuge de paix et une oasis de tranquillité. C'est le cas pour François Dumontier, traducteur aux Débats parlementaires, dans La Maison vide d'Harry Bernard :

Venu à Ottawa dès 1896, peu après l'avènement de Laurier, Dumontier avait été solliciteur d'assurances, journaliste au Temps et à la Justice, puis fonctionnaire du gouvernement. D'année en année, de patronage en patronage, il s'était hissé jusqu'au poste choisi de traducteur. Comme ses frères en fonctionnarisme, il menait une vie uniforme et satisfaite, sans préoccupations matérielles. [...] Il aimait son petit traintrain de vie, qui n'exigeait pas plus de travail épuisant que d'effort de pensée (Bernard, 1926 : $20 ; 26$ ).

D'autres personnages, en revanche, sont agacés d'être obligés de traduire, soit qu'ils ne se sentent pas à la hauteur de la tâche, soit que cette activité nuise à leurs aspirations d'écrivain. La secrétaire-traductrice Hélène Delongchamp dans Sylvie Stone, de Michel Beaulieu, est un exemple du premier cas. Elle se plaint de son sort en ces termes:

[...] j'aurais dû suivre le conseil de Sophie devenir mannequin les revues de modes les premières pages des magazines les voyages les nuits folles à courir les boites avec des hommes d'affaires alors que non j'échoue comme secrétaire-adjointe [sic] et on me demande en plus de traduire des textes parce que j'ai passé trois mois à New York [sic] il y a deux ans c'est vrai que j'ai dit trois ans plutôt que trois mois mais c'était pour les impressionner ça me tue ces traductions j'écris mal et je le sais que j'écris mal et je ne trouve jamais l'expression juste du premier coup il faut toujours que je demande à Alain et quand je me relis ça me désespère j'ai hâte que ça finisse qu'ils engagent donc du personnel d'autant plus qu'on ne m'explique jamais les attributions de secrétaire-adjointe - d'autant plus que tout le monde se sert de moi ici la belle poire [...] (Beaulieu, $1974:$ 106).

Pour sa part, le personnage anonyme créé par Renée-Berthe Drapeau dans N'entendre qu'un son exprime on ne peut plus clairement sa frustration d'avoir à produire des traductions pour l'agence "Les Traductions Clavier ", alors qu'elle aimerait se consacrer à l'écriture d'un roman : "Vous vous êtes enfermée dans votre petit bureau et vous avez constaté cette évidence que vous traduisiez les textes des autres "(Drapeau, $1988: 31$ ). Notre traductrice pensait sans doute au mot célèbre de Montesquieu, qui écrit dans ses Lettres persanes : "Si vous traduisez toujours, on ne vous traduira jamais " (Montesquieu, 1936 : 284). Si elle en était capable, cette traductrice anonyme du roman de Renée-Berthe Drapeau aimerait se traduire elle-même : "Vous auriez envie de reprendre votre roman à zéro et de le traduire comme vous le faites des articles sur l'artillerie américaine et la cigarette blonde chez Raoul Clavier " (Drapeau, $1988: 74)$. 
Havre de paix, source d'agacement, la traduction déclenche aussi des sentiments de frustration, voire d'agressivité. Dans Opération orchidée, Christiane Villon fait dire à son personnage Lucie, traductrice comme elle au Bureau de la traduction du gouvernement fédéral :

- Eh bien, on peut dire que, dans ton cas, le travail c'est la santé. Je ne pourrais pas en dire autant. Moi, je m'emmerde, je m'emmerde et je m'emmerde. On traduit en ce moment un rapport sur les pluies acides ; j'ai jamais rien vu d'aussi rébarbatif, je dirais même d'aussi caustique.

- C'est parce que tu n'es pas dans le bon bureau. Sais-tu pourquoi nous n'avons jamais trouvé les gens intéressants et spirituels que nous cherchions vainement au gouvernement? Souvienstoi, fonctionnaire était devenu synonyme de platitude (Villon, 1985 : 54).

Sa collègue traductrice, Sylvie, qui deviendra interprète et sera affectée à une mission d'espionnage pour le compte de la Gendarmerie royale du Canada, semble, elle aussi, se morfondre d'ennui devant son écran cathodique :

Elle termina rapidement la traduction des derniers paragraphes du rapport quil l'avait si peu intéressée qu'elle aurait été incapable de dire sur quoi il portait cinq minutes après y avoir mis le point final. Elle étira ses muscles douloureux et raidis par la tension de toute une journée passée au clavier d'un ordinateur (Villon, 1985 : 67).

Devenue interprète-espionne, elle aura l'impression d'avoir quitté " un monde peuplé de fantômes " pour entrer dans " un monde rempli d'ombres " (Villon, 1985 : 58).

\section{Érotisme et humour}

Contre toute attente, Jean-François Somcynsky évoque le métier d'interprète dans son roman érotique, Vingt minutes d'amour. L'action, si tant est que l'on puisse parler d'action, se déroule exclusivement entre les quatre murs d'une chambre d'hôtel où un homme et une femme qui ne se sont pas vus depuis huit mois font l'amour. Pendant leurs ébats, la belle laisse vagabonder ses pensées. Et à quoi pense-t-elle, croyez-vous ? À des cabines d'interprétation !...

N'ayant pas vraiment la force de se concentrer sur les événements [...], elle s'était mise à étudier la salle de conférence. Minutieusement. Tellement qu'elle pourrait encore la décrire. Le tableau, vert foncé, avec des mots, des bouts de phrase, des demi-cercles, des flèches, et les indications relatives à la traduction simultanée : Français 5 , Anglais 5 ('̀̀ l'arrière, les affiches sur les cabines indiquaient correctement : Français 1, Anglais 5. Elle n'avait pas mis les écouteurs, étant bilingue). (Somcynsky, 1983 : 55).

Présente dans les romans érotiques, la traduction l'est aussi dans les écrits de science-fiction, le plus souvent sous la forme de machines à traduire aux performances pour le moins étonnantes. Dans une nouvelle, "Oméga 8 est amoureux ", le même Jean-François Somcynsky invente un super cerveau qu'il baptise "Oméga 8 " : 
En trois microsecondes, il [0méga 8] engageait cinq cents personnes, en congédiait quatre cent soixante, explorait huit marchés, interprétait les mouvements de la bourse à Montréal, à New York et à Toronto, analysait les décisions de tous nos concurrents, [...] contrôlait les ventes, rationalisait la production, et traduisait les rapports de nos employés qui n'étaient pas encore bilingues (Somcynsky, 1981 : 92).

Et tout cela en trois microsecondes! Décidément, notre ami Somcynsky, qu'il donne dans l'érotisme ou la science-fiction, ne manque pas d'imagination ni d'humour... Devant la fabuleuse performance d'Oméga 8, la montre parlante capable de traduire et le logiciel de traduction automatique conçus par un ingénieur de l'École polytechnique de Montréal dans le roman de Monique LaRue (Copies conformes) paraissent des inventions bien anodines (LaRue 1989: 161). II en est de même de la machine à traduire portative qui livre des " équivalences culturelles ", dans une nouvelle d'Agnès Guitard, "Les virus ambiance " (Guitard, 1983 : 102).

\section{Conclusion}

Que peut-on conclure de ce survol des traducteurs présents dans la littérature québécoise? On peut sûrement affirmer que les personnages-traducteurs trahissent bel et bien les préoccupations des écrivains qui leur prêtent vie. II suffit d'ailleurs de lire les biographies ou autobiographies de ces auteurs pour se rendre compte de la concordance entre la vie romanesque et la vie réelle. Au cours d'une interview publiée dans Le Devoir, l'écrivain-enseignant François Gravel confiait à une journaliste : "Difficile d'oublier que je suis prof, même quand j'écris " (Bordeleau, 1991 : D-7). La plupart des auteurs qui ont mis en scène des traducteurs dans leurs romans ou leurs nouvelles pourraient affirmer à leur tour : "Difficile d'oublier que je suis traducteur, même quand j'écris. "

Les traducteurs sous lesquels se cachent les écrivains sont, dans une large mesure, les doubles de leurs créateurs qui se projettent dans leurs œuvres. Ce dédoublement s'effectue à la croisée de la fiction et de la réalité. C'est souvent dans les premiers romans, qui déguisent mal l'autobiographie, que l'on rencontre les traducteurs fictifs. Le spécialiste de Pierre Baillargeon, André Gaulin, est formel : "Sous Claude Perrin, c'est Pierre Baillargeon que nous retrouvons, les deux personnages se confondent " (Baillargeon, 1973). On pourrait en dire autant de nombreux autres créateurs de traducteurs imaginaires.

Entre la réalité et la fiction, on constate néanmoins quelques divergences importantes. On sait, par exemple, que la profession a un visage féminin. La proportion de femmes a toujours été supérieure à celle des hommes. Au début des années 1970, la STQ comptait environ $63 \%$ de femmes dans ses rangs. En 1986, ce pourcentage a grimpé à $78 \%$. En 2000, il se fixait à $68 \%$, chiffre identique à 1996. Donc, deux fois plus de femmes que d'hommes. Or, dans les œuvres littéraires québécoises, le rapport est inversé : on y dénombre deux fois plus de traducteurs que de traductrices. 
Autre différence de taille : les traducteurs fictifs semblent malheureux d'exercer la traduction. Ils se plaignent de leur sort, de leurs conditions de travail, de l'insipidité des textes qu'ils traduisent, de leur faible rémunération, etc. Bref, ils ne sont pas heureux. Les plus dépressifs se suicident. Les enquêtes menées auprès des membres de la STQ et de l'Ordre ces trente dernières années font entendre un tout autre son de cloche. En effet, sondage après sondage, les traducteurs " de la vraie vie " se déclarent, eux, satisfaits de leur sort dans une proportion supérieure à 80 \%. Ce pourcentage atteint même $86 \%$ en 1977 et en 1982. Des coqs en pâte, les traducteurs et les traductrices? Sans doute. Bizarrement, les derniers sondages ne posent plus la question...

Le profil du membre type en 1986 était le suivant : " II s'agit d'une femme d'environ 35 ans, traductrice agréée, possédant environ cinq années d'expérience comme professionnelle de la langue. Elle est détentrice d'un baccalauréat ou d'une licence, n'a pas fait d'études dans un domaine non linguistique et travaille comme salariée dans une entreprise privée où elle traduit en français des textes anglais. Cette spécialiste de la langue s'estime équitablement rétribuée pour les 37 heures que compte sa semaine normale de travail, jouit de bonnes conditions de travail et produit ses traductions sur un clavier-écran " (Delisle, 1990 : 328). En 2000, le profil socio-économique du membre type de l'OTTIAQ ressemblait plutôt à celui-ci : il s'agit toujours d'une femme, mais elle a maintenant 45 ans. Traductrice agréée, elle possède près de 15 ans d'expérience, détient un baccalauréat en traduction, travaille en pratique privée et traduit principalement du français vers l'anglais. Elle gagne en moyenne 50000 \$ par année (Sondage des services aux membres, 2000). Ce que ce sondage ne dit pas, c'est que cette travailleuse autonome travaille sûrement plus de 37 heures par semaine et que, malgré son poste de travail bien garni d'aides à la traduction, elle ne peut toujours pas satisfaire les échéances irréalistes (travaux exigés pour la veille). Est-elle heureuse ? C'est à elle de répondre, pensent les sondeurs.

Enfin, sur un plan plus général, on peut se demander si les très nombreux traducteurs fictifs qui peuplent la littérature québécoise ne sont pas aussi d'une certaine façon le miroir de l'imaginaire collectif québécois dans ses rapports avec l'Autre. On attend du traducteur qu'il soit le " serviteur de deux maîtres ", qu'il s'astreigne à une double fidélité : fidélité à l'auteur traduit, fidélité aux lecteurs. Cette double allégeance, replacée dans le contexte socio-politique canadien, ne pourrait-elle pas revêtir une valeur symbolique particulière ? Cette prolifération de traducteurs fictifs dans la littérature québécoise est-elle uniquement le reflet de la situation socio-économique de l'écrivain ? Ne pourrait-elle pas aussi être un questionnement sur les rapports entre francophones et anglophones au pays ? Un lieu de cristallisation de tensions larvées entre ces deux groupes linguistiques?

Mis à part les deux différences majeures entre les traducteurs fictifs et réels (répartition entre les sexes et degré de satisfaction professionnelle), les écrivains québécois ayant donné vie à des traducteurs imaginaires projettent une image assez fidèle, somme toute, du traducteur qué- 
bécois. Ils pourraient reprendre à leur compte cette réflexion de Julien Green : " J'éprouvai une satisfaction nouvelle, qui était d'avoir introduit dans un livre des personnages dont la réalité était indiscutable " (Green, 1987 : 237). La fiction, au fond, ce n'est pas le mensonge, c'est mentir pour dire vrai.

jdelisle@uOttawa.ca

Jean Delisle, trad. a., term. a, est diplômé de la Sorbonne Nouvelle (Paris III) et professeur émérite de I'Université d'Ottawa, où il a fait carrière de 1974 à 2007. Auteur et coauteur d'une vingtaine d'ouvrages, il a été traduit dans une quinzaine de langues. La pédagogie et l'histoire de la traduction sont ses deux champs de spécialisation.

\section{Bibliographie}

AGNANT, Marie-Célie (2001), Le Livre d'Emma, Montréal, Les Éditions du remue-ménage, Port-au-Prince, Les Éditions Mémoire, 167 p.

ANDRÈS, Bernard (1990), Écrire le Québec : de la contrainte à la contrariété, Montréal, XYZ éditeur, coll. "Études et documents ", 225 p.

BAILLARGEON, Pierre (1973), Les Médisances de Claude Perrin. Présenté par André Gaulin, Montréal, Les Éditions du Jour, 197 p. [Montréal, Parizeau, 1945].

BAILLARGEON, Pierre (1944), "La carrière des lettres ", dans L'Amérique française, $n^{\circ} 21$, p. 48-56.

BEAULIEU, Michel (1974), Sylvie Stone, Montréal, Les Éditions du Jour, 177 p. [Écrit en 1969] BELLEAU, André (1980), Le Romancier fictif, Québec, Les Presses de l'Université du Québec, $155 \mathrm{p}$.

BERNARD, Harry (1926), La Maison vide, Montréal, Bibliothèque de l'Action française, 203 p. BLAIS, Marie-Claire (1980), Une Liaison parisienne, Postface critique de François Ricard, Montréal, Les Quinze éditeur, $181 \mathrm{p}$.

BORDELEAU, Francine (1991), "François Gravel. Les contes d'un menteur professionnel ", dans Le Devoir, 19 octobre, p. D-7.

DELISLE, Jean (1990), Les Alchimistes des langues, Ottawa, Les Presses de l'Université d'Ottawa, $446 \mathrm{p}$.

DELISLE, Jean (1999), Portraits de traducteurs, Ottawa, Les Presses de l'Université d'Ottawa, $305 p$. 
DRAPEAU, Renée-Berthe (1988), N'entendre qu'un son, Montréal, L'Hexagone, 109 p. ÉLIE, Robert (1950), La Fin des songes, Montréal, éditions Beauchemin, 256 p. GÉRIN-LAJOIE, Antoine (1947), Jean Rivard, Montréal, Librairie Beauchemin, 292 p. [Québec, Les Soirées canadiennes, vol. 2, 1862]

GREEN, Julien (1987), Le Langage et son double, Paris, éditions du Seuil, coll. "Points ", $n^{\circ} 190,403 \mathrm{p}$.

GUAY, Jean-Pierre (1983), Lorsque notre littérature était jeune. Propos de Pierre Tisseyre recueillis et présentés par J.-P. Guay, Montréal, Le Cercle du Livre de France, 264 p.

GUITARD, Agnès (1983), "Les virus ambiance ", dans Les Années-lumière. Dix nouvelles de science-fiction réunies et présentées par Jean-Marc Gouanvic, Montréal, Vlb éditeur, p. 77-127. LAPIERRE, René (1983), Comme des mannequins, Montréal, Les Éditions Primeur, 176 p.

LARUE, Monique (1989), Copies conformes, Montréal, Les Éditions Lacombe, 190 p.

LAVIGNE, Nicole (1998), Moscou la nuit, Montréal, Éditions des Intouchables, 197 p.

MAUREL, Charles (1946), " Nos héros de romans ", dans Canada français, sept. 1945, p. 43-45, janv. 1946, p. 263-270.

MONTESQUIEU, Charles-Louis de Secondat (1936), Les Lettres persanes, Publiées avec une introduction par Jacques Bainville, Paris, La cité des livres, Lettre 128, p. 282-285. [Première édition : 1721]

POULIN, Jacques (1978), Les Grandes Marées, Montréal, Leméac, 201 p.

SAINT-MARTIN, Lori (1991), Lettre imaginaire à la femme de mon amant, Montréal, L'Hexagone, $133 \mathrm{p.}$

SOMCYNSKY, Jean-François (1981), "Oméga 8 est amoureux ", dans Peut-être à Tokyo, Sherbrooke, Éditions Naaman, p. 90-103. [Écrit en 1966]

SOMCYNSKY, Jean-François (1983), Vingt minutes d'amour, Montréal, Le Cercle du Livre de France, 100 p. [Écrit en 1978]

TATILON, Claude (1991), Hélèna, Paris, Éditions de l'Arcantère, 133 p.

VILLON, Christiane (1985), Opération orchidée, Montréal, Le Jour, éditeur, 187 p. 